\title{
Evanescent states in quantum wires with Rashba spin-orbit coupling
}

\author{
Llorenç Serra, ${ }^{1,2}$ David Sánchez, ${ }^{1}$ and Rosa López ${ }^{1}$ \\ ${ }^{1}$ Departament de Física, Universitat de les Illes Balears, E-07122 Palma de Mallorca, Spain \\ ${ }^{2}$ Institut Mediterrani d'Estudis Avançats IMEDEA (CSIC-UIB), E-07122 Palma de Mallorca, Spain
}

(Received 25 January 2007; revised manuscript received 20 April 2007; published 31 July 2007)

\begin{abstract}
We discuss the calculation of evanescent states in quasi-one-dimensional quantum wires in the presence of Rashba spin-orbit interaction. We suggest a computational algorithm devised for cases in which longitudinal and transverse motions are coupled. The dispersion relations are given for some selected cases, illustrating the feasibility of the proposed computational method. As a practical application, we discuss the solutions for a wire containing a potential step.
\end{abstract}

DOI: 10.1103/PhysRevB.76.045339

PACS number(s): 73.63.Nm

\section{INTRODUCTION}

Quantum wires formed in two-dimensional electron gases are electron waveguides allowing transmission only along one direction. Due to the lateral confinement, at typical electron densities a number of energy subbands may be occupied, which are referred to as transverse modes or channels. Depending on the behavior of the electronic states along the wire they are usually classified as propagating and evanescent. The latter decay with the distance and, therefore, are irrelevant in the asymptotic regions of the quantum wire, where only propagating modes can exist. Nevertheless, evanescent modes are of paramount importance for a wire with inhomogeneities such as potential barriers or wells. ${ }^{1}$ This is because the evanescent modes strongly influence the scattering conditions determining the reflection and transmission coefficients from a given potential inhomogeneity. This way, the evanescent modes determine the amplitudes of the propagating modes in the asymptotic regions of a wire with a scattering center.

The wave number $k$ gives the dependence with the distance $x$ along the wire for each specific mode as $\exp (i k x)$. While for propagating modes $k$ is a real number, for evanescent states it must obviously have an imaginary part. In the most general case $k$ may also contain a real part for evanescent modes. When transverse and longitudinal motions are decoupled the wave numbers for propagating and evanescent modes can be trivially obtained. Indeed, if transverse motion energies are quantized by a set of $k$-independent eigenvalues $\varepsilon_{n}$, with $n=1,2, \ldots$, the propagating mode wave numbers fulfill

$$
k= \pm \sqrt{2 m\left(E-\varepsilon_{n}\right)} / \hbar \quad\left(E \geqslant \varepsilon_{n}\right),
$$

where $E$ is the total energy. On the contrary, evanescent modes are characterized by

$$
k= \pm i \sqrt{2 m\left(\varepsilon_{n}-E\right)} / \hbar \quad\left(E<\varepsilon_{n}\right) .
$$

The above scenario of Eqs. (1) and (2) with analytically known wave numbers, purely real and purely imaginary for propagating and evanescent modes, respectively, breaks down when motion along the longitudinal and transverse directions are coupled. In this case, transverse quantization is not given by a fixed set of eigenvalues $\varepsilon_{n}$ but must be solved explicitly for each $E$ and $k$. This makes, in practice, the cal- culation of evanescent states a formidable task. The mathematical difficulty is exacerbated by the fact that for general complex $k$ 's one loses standard properties such as the Hermitian condition of the eigenvalue equation, required in most matrix diagonalization algorithms. As a matter of fact, given $E$ the problem can be mathematically presented as a nonlinear eigenvalue equation for $k$. This situation is found, for instance, when a magnetic field is coupled with the electron's orbital motion ${ }^{2}$ or in the specific case addressed in this work, when a spin-orbit coupling is present. Evanescent states with spin-orbit interaction have been recently considered in Refs. 3 and 4 in the context of plane-wave formalisms, adequate for interfaces between flat bottom potentials. However, to the best of our knowledge, a general method to obtain the evanescent modes in transmission channels with arbitrary transverse confinements is lacking in the literature.

We stress that the evanescent states do not fulfill proper boundary conditions along the channel since the fact that they vanish in one direction necessarily implies their divergence in the reversed one. These modes are, therefore, not physically realizable in the entire channel, for $x$ spanning the interval $(-\infty,+\infty)$. Nevertheless, they are extremely important because many physical states do behave as evanescent in restricted domains. This unphysical condition of the extended evanescent states explains why, mathematically, they are solutions of a non-Hermitian problem, while physical states always originate from Hermitian operators in quantum mechanics.

In this work we shall discuss a practical algorithm to obtain the evanescent modes when transverse and longitudinal motions are coupled through a spin-orbit term of Rashba type, ${ }^{5}$ typical of two-dimensional electron nanostructures. Although in the specific applications we shall assume a parabolic transverse potential, the suggested approach will be equally valid for arbitrary transverse confinements. Numerical results for selected values of the Rashba coupling strength will be presented as an illustration of the method. Interest in the Rashba spin-orbit coupling is mostly due to the tunability of the Rashba strength by means of electric gates which opens a possibility of spin control in nanostructures, with the Datta and Das spin transistor as a well-known spintronic device proposal. ${ }^{6}$

This paper is organized as follows. In Sec. II we present the physical system. Section III is devoted to the practical 
algorithm to compute evanescent states and Sec. IV shows selected numerical results and a practical application of our approach. Finally, Sec. V draws the conclusions of the work.

\section{PHYSICAL SYSTEM}

We consider a two-dimensional electron gas lying on the $x y$ plane, with a parabolic confinement in the $y$ direction and perfect translational invariance along $x$. The corresponding Hamiltonian operator $\mathcal{H}_{0}$ reads

$$
\mathcal{H}_{0}=\frac{p_{x}^{2}+p_{y}^{2}}{2 m}+\frac{1}{2} m \omega_{0}^{2} y^{2} .
$$

Additionally, a spin-orbit coupling of Rashba type, with coupling strength $\alpha$, is also active,

$$
\mathcal{H}_{R}=\frac{\alpha}{\hbar}\left(p_{y} \sigma_{x}-p_{x} \sigma_{y}\right) .
$$

The coupling strength $\alpha$ is assumed constant throughout the system, including the asymptotic regions in the $x$ and $y$ directions. The total Hamiltonian is thus $\mathcal{H}=\mathcal{H}_{0}+\mathcal{H}_{R}$ and we are interested in the solutions of Schrödinger's equation for a given energy $E$,

$$
(\mathcal{H}-E) \Psi(x, y, \eta)=0,
$$

where $\eta=\uparrow, \downarrow$ is labeling the spin double valued variable. Any energy is physically acceptable since the wire spectrum will be continuous for the propagating modes and, moreover, since we also consider evanescent modes even negative energies will yield solutions to Eq. (5).

As mentioned in the Introduction, the spinorial wave function is assumed to be separable in the following form:

$$
\Psi(x, y, \eta) \equiv \phi(y, \eta) e^{i k x} .
$$

It is convenient to express the spin-dependent part in terms of the eigenspinors of $\sigma_{x}, \chi_{x \pm}(\eta)$, as

$$
\phi(y, \eta) \equiv \phi_{1}(y) \chi_{x+}(\eta)+\phi_{2}(y) \chi_{x-}(\eta) .
$$

Equation (5) can be recast as a matrix equation for the amplitudes $\phi_{1,2}(y)$,

$$
\left(\begin{array}{cc}
h_{0}-i \alpha \frac{d}{d y}+\frac{\hbar^{2} k^{2}}{2 m}-E & -i \alpha k \\
i \alpha k & h_{0}+i \alpha \frac{d}{d y}+\frac{\hbar^{2} k^{2}}{2 m}-E
\end{array}\right)\left(\begin{array}{l}
\phi_{1}(y) \\
\phi_{2}(y)
\end{array}\right)
$$

where we have defined the transverse oscillator operator

$$
h_{0} \equiv-\frac{\hbar^{2}}{2 m} \frac{d^{2}}{d y^{2}}+\frac{1}{2} m \omega_{0}^{2} y^{2} .
$$

Equation (8) is the central equation we intend to solve in this work. Although it has the formal appearance of a linear eigenvalue equation, with eigenvalue $E$, in our case the energy is given and what is unknown is the wave number $k$. Notice also that for complex $k$ 's the matrix in Eq. (8) in non-
Hermitian, which prevents the use of standard matrix diagonalization routines. This invalidates the computational strategy normally used for propagating modes and consisting of: (a) preassign a value to $k$; (b) diagonalize Eq. (8); and (c) find a posteriori what $k$ 's give as eigenvalues the energy of interest $E$.

\section{ALGORITHM}

An essential property allowing a strategy to solve Eq. (8) is that the amplitudes $\phi_{1,2}(y)$ must fulfill the boundary condition that they vanish for $y \rightarrow \pm \infty$. Of course, $\phi_{1,2}(y)$ must also be smooth functions of $y$, continuous and with continuous derivatives for all $y$ 's. These conditions can be fulfilled at the same time only for some specific wave numbers representing propagating (real $k$ 's) and evanescent (complex $k$ 's) modes.

Our algorithm is based on the use of finite differences, discretizing the $y$ axis in $N$ uniformly distributed points in the interval $\left[y_{\min }, y_{\max }\right]$. The derivatives can then be computed using $n$-point formulas, ${ }^{7}$ i.e., $n$ neighboring points are required to compute the derivative at each grid point. We transform our problem into a linear system of $2 N$ scalar linear equations, yielding the $2 \mathrm{~N}$ unknowns corresponding to $\phi_{1}$ and $\phi_{2}$ on the grid. This linear system has a solution fulfilling the boundary conditions at $y_{\min }$ and $y_{\max }$ for any $k$, real or complex, but only for the physically acceptable wave numbers both functions have a continuous first derivative at an arbitrarily chosen matching point $y_{m}$. Since the derivatives may be discontinuous at $y_{m}$ it is essential in the calculation of the derivatives not to invoke points lying on opposite sides of the matching border. This is accomplished with the use of noncentered formulas for the derivatives at points near the matching border.

At the matching point we do not impose the discretized Eq. (8) but, instead, we ensure a solution different from the trivial one (vanishing for all $y$ 's) by arbitrarily choosing that $\phi_{1}\left(y_{m}\right)=1$. An additional equation is needed to have as many equations as unknowns. We impose continuity of the first derivative of $\phi_{2}$ at $y_{m}$. In summary, the resulting linear system is given by:

(a) $(2 N-2)$ equations obtained by discretizing Eq. (8) for $y \neq y_{m}$, without crossing the matching border for the derivatives;

(b) $\phi_{1}\left(y_{m}\right)=1$; and

(c) $\left(d \phi_{2} / d y\right)_{L}-\left(d \phi_{2} / d y\right)_{R}=0$, where $\left(d \phi_{2} / d y\right)_{L, R}$ represent $\phi_{2}$ derivatives at $y_{m}$ using noncentered formulas with the left $(L)$ and right $(R)$ neighboring points.

The suggested algorithm does not yield normalized transverse amplitudes but, of course, normalization $\int\left|\phi_{1}(y)\right|^{2}+\left|\phi_{2}(y)\right|^{2} d y=1$ can be trivially imposed after the solution has been obtained.

The matrix representing the linear system is greatly sparse and can be very efficiently solved using standard numerical routines. ${ }^{8}$ The precision of the method increases when using larger $n$ 's for the derivatives. We have used up to 11-point formulas but, typically, five or seven points already provide quite accurate results. As an example, Fig. 1 shows the amplitudes $\phi_{1,2}(y)$ for two different wave numbers: the upper 


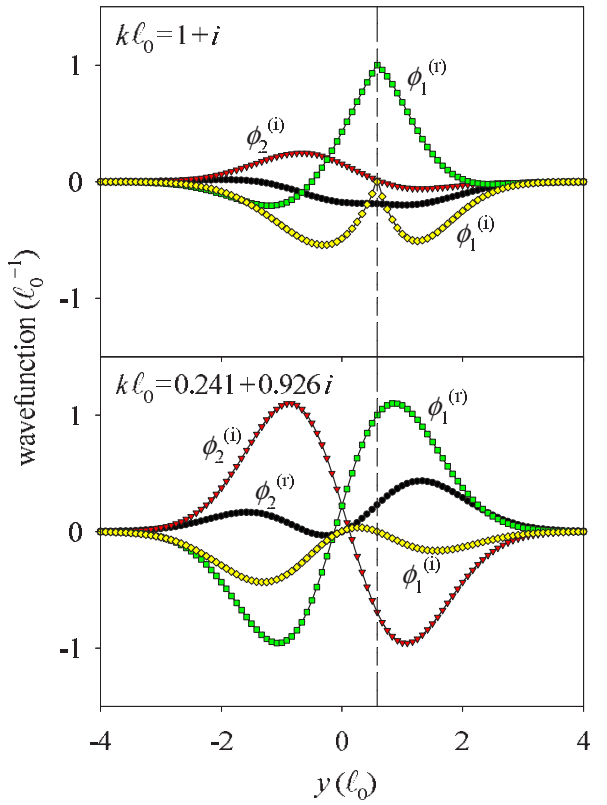

FIG. 1. (Color online) Wave-function amplitudes illustrating the algorithm. Real and imaginary parts correspond, respectively, to (r) and (i) superscripts. The total energy is $E=\hbar \omega_{0}$ while the Rashba strength is chosen as $\alpha=0.3 \hbar \omega_{0} \ell_{0}$, with $\ell_{0}=\sqrt{\hbar / m \omega_{0}}$ the oscillator length. The position of the matching point is given by the dashed line. Upper and lower panels correspond to the indicated wave numbers.

panel shows a discontinuous first derivative at the matching point, indicating that the chosen $k$ is not physically valid; the situation is different in the lower panel, with perfectly smooth functions.

Finding the physically acceptable wave numbers is done by exploring the complex- $k$ plane. In practice, we define the real function

$$
\mathcal{F}(k)=\left|\left(\frac{d \phi_{1}\left(y_{m}\right)}{d y}\right)_{L}-\left(\frac{d \phi_{1}\left(y_{m}\right)}{d y}\right)_{R}\right|,
$$

and look for the zeros of $\mathcal{F}$ by sweeping $\operatorname{Re}(k)$ and $\operatorname{Im}(k)$, the real and imaginary parts of the wave number, within a preselected range.

\section{RESULTS}

\section{A. Symmetry considerations}

Figure 2 shows the contour lines of $\mathcal{F}$ circling the position of the nodes for selected values of $E$ and $\alpha$. Having determined the approximate locations, it is then a simple matter to zoom in and accurately determine the nodes. One must be careful, however, not to miss nodes for which the corresponding minimum is very narrow. The figure illustrates a symmetry in the $k$ plane: if a mode has a certain $k$ then wave numbers obtained by changing $\operatorname{sign} \pm \operatorname{Re}(k)$ and/or $\pm \operatorname{Im}(k)$ are also physically valid. Similar conditions were discussed in Ref. 2 for a quantum wire in a perpendicular magnetic field. Evanescent modes with wave numbers having a nonzero real part will therefore come in groups of four, such as

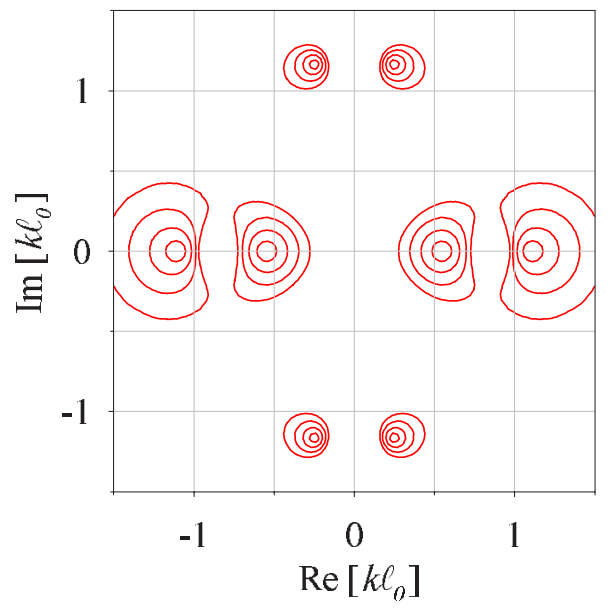

FIG. 2. (Color online) Contour lines of $\mathcal{F}(k)$ showing the position of the nodes. We have used $E=0.75 \hbar \omega_{0}$ and $\alpha=0.3 \hbar \omega_{0} \ell_{0}$.

the modes in Fig. 2 with $k= \pm 0.247 \pm 1.166 i$, corresponding to $k, k^{*},-k$, and $-k^{*}$.

Mode degeneracies can be explained taking into account that if a solution to Eq. (8) is characterized by a given wave number and amplitudes $\left\{k, \phi_{1}, \phi_{2}\right\}$ then, by simply taking the complex conjugate of Eq. (8), we find a solution having $\left\{k^{*}, \phi_{2}^{*}, \phi_{1}^{*}\right\}$. On the other hand, our starting Hamiltonian $\mathcal{H}$ is time reversal invariant which, for a spin $1 / 2$ system, implies that the solutions must appear in degenerate pairs of time reversed states known as Kramers doublets. Using the time reversal operator for a spin $1 / 2$ system, $\Theta=-i \sigma_{y} \mathcal{K}$, where $\mathcal{K}$ represents complex conjugation, we find the Kramers partner of $\left\{k, \phi_{1}, \phi_{2}\right\}$ as $\left\{-k^{*}, \phi_{2}^{*},-\phi_{1}^{*}\right\}$. In summary, with both complex conjugation (c.c.) and time reversal symmetry we obtain the fourfold degeneracy

$$
\begin{array}{cccc}
\left\{k, \phi_{1}, \phi_{2}\right\} & \text { c.c. } & \left\{k^{*}, \phi_{2}^{*}, \phi_{1}^{*}\right\} \\
\Theta \uparrow & \leftrightarrow & -\Theta \uparrow \\
\left\{-k^{*}, \phi_{2}^{*},-\phi_{1}^{*}\right\} & \stackrel{\text { c.c. }}{\leftrightarrow} & \left\{-k,-\phi_{1}, \phi_{2}\right\} .
\end{array}
$$

It is worth stressing that the degeneracies expressed by Eq. (11) are valid irrespective of the $y$-inversion symmetry of the wire potential. This is a qualitative difference with the result for wires in perpendicular magnetic field, ${ }^{2}$ where it was found that asymmetric wires display asymmetric $\operatorname{Re}(k)$ branches, i.e., they do not fulfill the symmetry $k \leftrightarrow-k$. The difference originates from the breaking of time reversal invariance by the external magnetic field.

\section{B. Mode dispersion diagrams}

The mode dispersion for the same wire of the preceding figures is shown in Fig. 3. We only plot positive wave numbers noting that, with the above-mentioned symmetry, wavenumber signs can be inverted giving a fourfold degeneracy of each evanescent mode and twofold for the propagating ones. The dispersion of propagating modes shows a familiar picture, already investigated in detail. ${ }^{9-14}$ There is a threshold energy $E_{n}^{(t h)}$ for the activation of the $n$th propagating mode. 


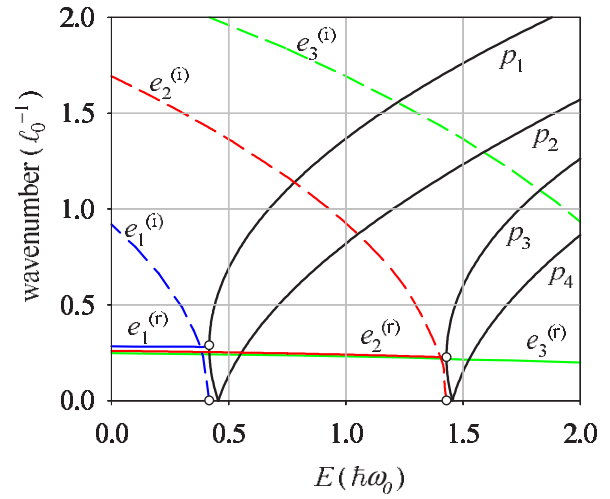

FIG. 3. (Color online) Mode dispersion for $\alpha=0.3 \hbar \omega_{0} \ell_{0}$. Branch labels indicate mode number $n$ as well as evanescent or propagating character as $e_{n}$ and $p_{n}$, respectively. In the case of evanescent modes, superindexes $(r)$ and $(i)$ are used to indicate whether the branch gives the real or the imaginary part of the wave number.

Due to the spin-orbit coupling, when $E$ slightly exceeds $E_{n}^{(t h)}$, propagating states with nonzero wave number $k_{n}^{(t h)}$, belonging to the $n$th mode, are allowed.

Surprisingly, the evanescent modes in Fig. 3 resemble those of asymmetric wires in vertical magnetic field discussed in Ref. 2, in spite of the present wires being symmetric and having no field. For $E$ slightly below $E_{n}^{(t h)}$ there is an evanescent mode whose $\operatorname{Im}(k)$ approaches zero as $E$ approaches $E_{n}^{(t h)}$ from below but, quite remarkably, $\operatorname{Re}(k)$ remains essentially stuck at the threshold value for the propagating mode, $k_{n}^{(t h)}$. This is true even for energies much smaller than $E_{n}^{(t h)^{2}}$. Notice that, on the contrary, for evanescent states $\operatorname{Im}(k)$ rapidly increases when $E$ decreases, indicating a faster decay of the evanescent mode when the energy separates from the threshold $E_{n}^{(t h)}$. Strictly speaking, the real part also varies with $E$ although it can be hardly noticed to the scale of Fig. 3.

Figure 4 shows the mode dispersion for a case of strong spin-orbit coupling, when $\alpha=\hbar \omega_{0} \ell_{0}$. As compared to the weak coupling case (Fig. 3), the mode branch distribution now looks more complicated. Nevertheless, it remains true that below the threshold of a propagating mode there is an evanescent mode whose wave number initially coincides

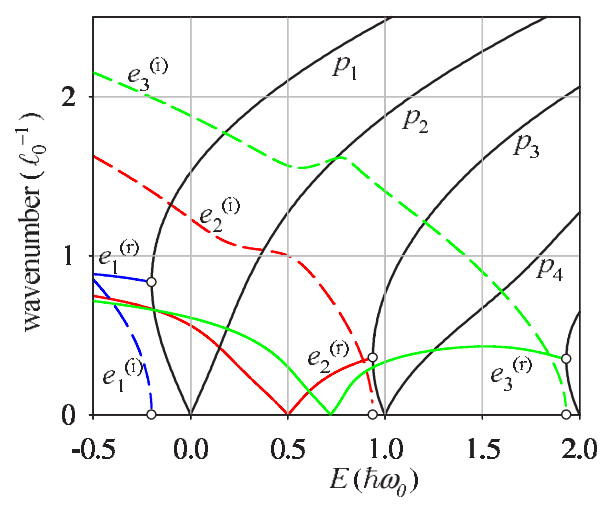

FIG. 4. (Color online) Same as Fig. 3 for $\alpha=\hbar \omega_{0} \ell_{0}$. with $k_{n}^{(t h)}$, the wave number of the propagating mode at threshold. If the energy is further decreased, $\operatorname{Re}(k)$ does not remain almost constant as in Fig. 3, but displays a rather strong variation, first decreasing and then increasing again. It is worth stressing that the symmetry discussed above still applies to Fig. 4 and one can obtain valid wave numbers by reverting signs in either $\operatorname{Re}(k)$ or $\operatorname{Im}(k)$. Another conspicuous feature of Fig. 4 is that $e_{n}^{(i)}$, the branches giving $\operatorname{Im}(k)$, are not monotonously increasing with decreasing energy but display a marked wiggle at $E \sim 0.4 \hbar \omega_{0}$ and $0.6 \hbar \omega_{0}$ for $e_{2}^{(i)}$ and $e_{3}^{(i)}$, respectively.

The simpler behavior of the weak coupling case suggests an analytical expression for the evanescent mode wave numbers. We have indeed found that when $\alpha /\left(\hbar \omega_{0} \ell_{0}\right) \ll 1$ the evanescent mode wave numbers can be well-approximated by

$$
k= \pm k_{n}^{(t h)} \pm i \sqrt{2 m\left(E_{n}^{(t h)}-E\right)} / \hbar \quad\left(E<E_{n}^{(t h)}\right),
$$

in terms of the threshold energy $E_{n}^{(t h)}$ and wave number $k_{n}^{(t h)}$ of the $n$th propagating mode. Noting now that in the considered limit one has $k_{n}^{(t h)} \approx k_{R}$ and $E_{n}^{(t h)} \approx \varepsilon_{n}$, where we define the Rashba wave number $k_{R}=\alpha m / \hbar^{2}$ and the transverse oscillator energies $\varepsilon_{n}=(n+1 / 2) \hbar \omega_{0}$, we can further simplify Eq. (12) to

$$
k= \pm k_{R} \pm i \sqrt{2 m\left(\varepsilon_{n}-E\right)} / \hbar \quad\left(E<\varepsilon_{n}\right) .
$$

This is a very appealing result, stating that in the weak coupling case the only modification to Eq. (2) introduced by the Rashba coupling is to add a constant (energy independent) real part given by the Rashba wave number. This result agrees with the findings of Ref. 4, where the density oscillations at the interface between a ferromagnet and a semiconductor with Rashba interaction were found to have an energy independent wavelength $\lambda \sim k_{R}^{-1}$ in the limit of low Rashba coupling.

Equation (13) can be understood noting that in the weak spin-orbit limit one can neglect the Rashba intersubband coupling term, proportional to $p_{y} \sigma_{x}$. In this approximation the Hamiltonian is diagonal in the $\sigma_{y}$ basis and the corresponding Schrödinger's equation for any $k$ implies

$$
\varepsilon_{n}+\frac{\hbar^{2} k^{2}}{2 m} \pm \frac{\hbar^{2} k_{R} k}{m}-E=0
$$

whose solutions for $E<\varepsilon_{n}$ and neglecting terms in $k_{R}^{2}$ coincide with Eq. (13).

\section{Potential step}

To illustrate the use of the computed evanescent modes with a specific example we have obtained the wave functions in a quantum wire with Rashba interaction containing a potential step $V_{0} \Theta(x) .{ }^{15}$ Electrons incident from the left $(x$ $<0$ ) impinge on the step border and, assuming their energy is not enough to allow for propagation in the right region $(x>0)$, only pure evanescent modes will be seen for $x>0$. On the contrary, the left region will contain three types of modes: incident propagating, reflected propagating, and eva- 


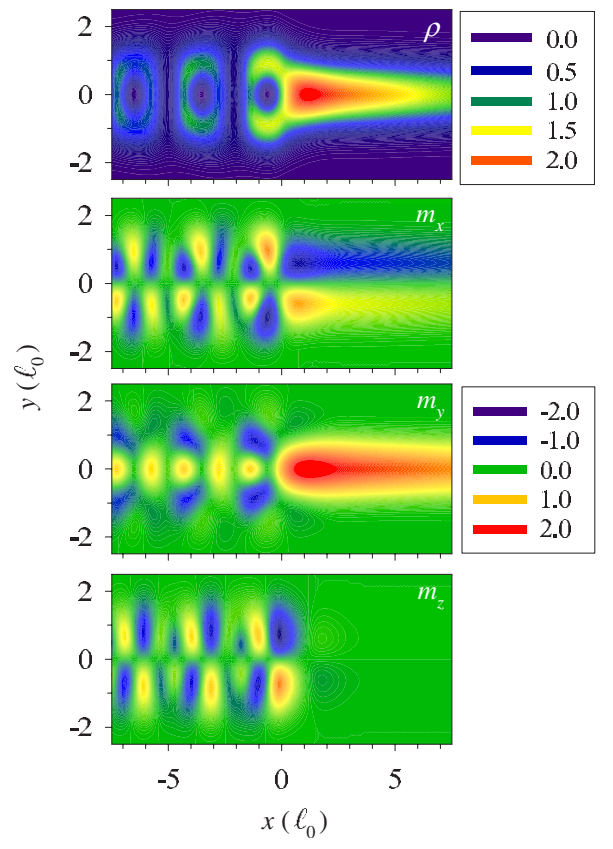

FIG. 5. (Color online) Density and spin magnetization distributions in a wire with a potential step at $x=0$ of $V_{0}=1.13 \hbar \omega_{0}$ and having $\alpha=\hbar \omega_{0} \ell_{0}$. The density contour numerical values are given in units of $\ell_{0}^{-2}$ while those of magnetization density are in units of $\hbar /\left(2 \ell_{0}^{2}\right)$. Incidence is from the left in mode $p_{1}$ shown in Fig. 4 , for an energy $E=0.93 \hbar \omega_{0}$ slightly below the threshold for propagating modes $p_{3}$ and $p_{4}$.

nescent. The mode wave numbers before and after the step can be obtained from the mode dispersion diagrams, Figs. 3 and 4 , associating to each region an energy measured from the potential bottom, i.e., the left $E_{l}=E$ and right $E_{r}=E-V_{0}$ energies. At the step edge the wave functions must match adequately, this condition determining the amplitudes of the reflected propagating modes for $x<0$ and of the evanescent waves for all $x$. The Appendix details the resulting equations and their practical resolution method. Evanescent states are crucial in this problem for without them it is not possible to fulfill the matching conditions at $x=0$ and for any value of the transverse coordinate $y$ (see Appendix). Besides, evanescent states must be considered in order to satisfy the obvious requirement that the reflected flux coincides with the incident one.

Figure 5 displays the density and spin magnetizations obtained after solving the linear system of equations corresponding to the matching conditions. We have assumed the same intense Rashba coupling $\alpha=\hbar \omega_{0} \ell_{0}$ of Fig. 4, and a left incidence of unit flux from mode $p_{1}$, with energy $E$ $=0.93 \hbar \omega_{0}$, slightly below the propagation threshold for modes $p_{3}$ and $p_{4}$. The step is chosen as $V_{0}=1.13 \hbar \omega_{0} \ell_{0}$, which implies that the wave numbers are those of Fig. 4 at energies $\left(E_{l}, E_{r}\right)=(0.93,-0.20) \hbar \omega_{0}$. The spatial distribution of density and magnetization due to evanescent modes are clearly seen for $x>0$, while on the left side there are marked interference effects between the coexisting modes. Actually, the latter patterns are similar to those discussed in Ref. 4 when considering a different physical system; namely, an interface between a ferromagnet and a semiconductor. Focus-

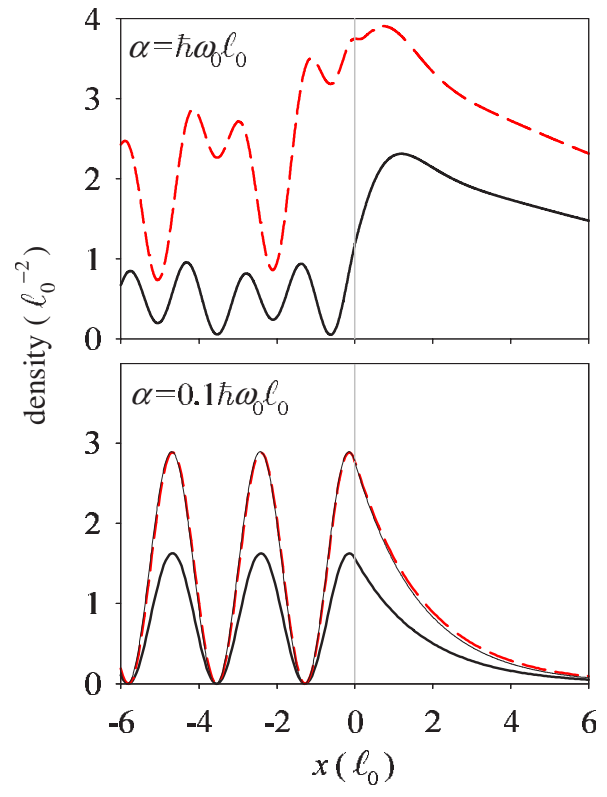

FIG. 6. (Color online) Density along the $y=0$ cut (thick solid line) and integrated in $y, \int_{-\infty}^{\infty} d y \rho(y) / \ell_{0}$ (dashed line). The upper panel corresponds to the solution given in the upper panel of Fig. 5. The lower panel corresponds to a weaker Rashba coupling, with parameters $\alpha=0.1 \hbar \omega_{0} \ell_{0}$ and $\left(E_{l}, E_{r}\right)=(1.45,0.45) \hbar \omega_{0}$, corresponding to an energy $E=1.45 \hbar \omega_{0}$ and step height $V_{0}=\hbar \omega_{0}$. The thin solid line of the lower panel, almost superimposing on the dashed line, corresponds to the strict 1D problem.

ing on the evanescent mode side, there is a strong injection of spin $y$ magnetization $m_{y}$, clearly due to the fact that the incident mode $p_{1}$ is mostly polarized along $+y$ (it is not completely polarized due to the admixture induced by the Rashba intersubband coupling term $\left.\alpha p_{y} \sigma_{x}\right)$. There is also an important accumulation of evanescent $m_{x}$ magnetization of different signs on the two sides of the wire. Had we considered incidence from mode $p_{2}$ at the same energy, the spin magnetizations would be reverted with respect to those of Fig. 5, indicating that the average for incidence from the two modes $p_{1}$ and $p_{2}$ does not produce any net magnetization, not even locally. On the contrary, the density shown in the upper panel of Fig. 5 is the same for incidence from any of the two modes.

The vanishing spin magnetization when adding the contributions from the two left incident channels $p_{1}$ and $p_{2}$ can be interpreted, theoretically, as a manifestation of the time reversal symmetry which is conserved by the Rashba interaction. Indeed, when $E_{r}$ is such that there can be no propagation to the right all incident flux is reflected backwards, yielding a solution which is invariant by time reversal.

A remarkable feature in Fig. 5 is that the density takes its maximum value for $x>0$, the evanescent mode side. This is totally unexpected for purely exponentially decaying modes. However, since the evanescent wave numbers with Rashba coupling are complex, we may find a superposition of oscillating and exponentially decaying distributions, as that of Fig. 5. This behavior is a peculiarity of intense Rashba couplings. To further clarify this we plot in Fig. 6 the densities for weak and strong values of $\alpha$. In the weak $\alpha$ case the 


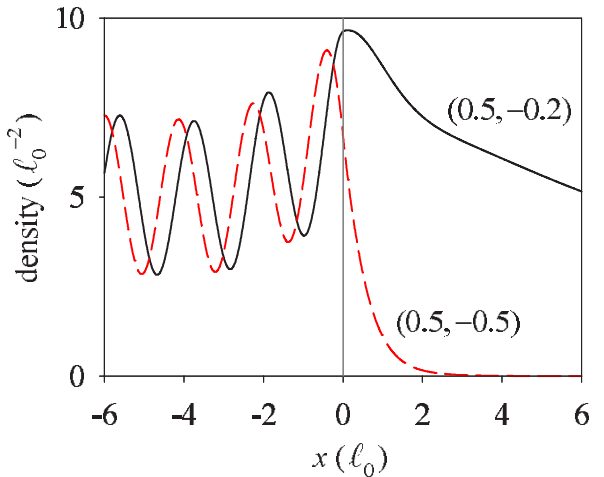

FIG. 7. (Color online) $y$-integrated densities $\int_{-\infty}^{\infty} d y \rho(y) / \ell_{0}$ for the shown values of $\left(E_{l}, E_{r}\right)$ in $\hbar \omega_{0}$ units for the strong coupling limit $\alpha=\hbar \omega_{0} \ell_{0}$. Left incidence of unit flux from modes $p_{1}$ and $p_{2}$ is assumed.

evanescent density is indeed given by a pure exponential decay that can be explained taking the wave numbers given by Eq. (13). Since $\operatorname{Re}(k)$ is constant, it amounts to a common phase for all modes and thus is irrelevant for the density, remaining only the pure exponentially decaying contributions. The lower panel also shows the density in the strict 1D limit, when all transverse motion is neglected and the Hamiltonian reduces to $\mathcal{H}_{1 D}=p_{x}^{2} / 2 m-\alpha p_{x} \sigma_{y} / \hbar$. The excellent agreement with the $y$-integrated $2 \mathrm{D}$ density clearly proves that in the limit of small $\alpha$ the problem becomes effectively one-dimensional, as should be expected.

The relevance of the evanescent modes in the strong $\alpha$ case manifests itself in Fig. 6 as conspicuous accumulations of the integrated density (dashed line), slowly decaying towards both sides of the edge. The decay is quite sensitive to the values of $E$ and $V_{0}$ or, equivalently, to the energies $\left(E_{l}, E_{r}\right)$ fixing the left and right modes. This is clearly seen in Fig. 7, which displays the $y$-integrated densities for $\left(E_{l}, E_{r}\right)$ $=(0.5,-0.2) \hbar \omega_{0}$ and $(0.5,-0.5) \hbar \omega_{0}$. Towards the left the decay is now faster than in the upper panel of Fig. 6, due to the higher values of $\operatorname{Im}(k)$ for this $E_{l}$. The same argument explains the faster decay towards the right for $E_{r}=-0.5 \hbar \omega_{0}$. In general, we find that $E_{l}$ and $E_{r}$ determine the decay rate towards the left and right sides rather independently.

Most interestingly, the evanescent modes are crucial even when the energy $E_{r}$ exceeds the propagation threshold to the right of the step. This is shown in Fig. 8, where the total transmission for left incidence in both modes $p_{1}$ and $p_{2}$ is given for the strong coupling case. We have fixed $E_{l}$ $=0.93 \hbar \omega_{0} \ell_{0}$ and increased $E_{r}$ above the propagation threshold of modes $p_{1}$ and $p_{2}$. In practice, this corresponds to lowering the step height $V_{0}=E_{l}-E_{r}$ while keeping fixed the incident energy $E=E_{l}$. The triangles are the result when evanescent modes are totally neglected in subsets $\left\{\widetilde{\phi}_{n}, \widetilde{k}_{n}\right\}$ and $\left\{\hat{\phi}_{n}, \hat{k}_{n}\right\}$ of the Appendix. There are sizeable differences with the transmission obtained when including enough evanescent states in both subsets (circles). Quite remarkably, the condition of flux conservation which, for the present situation, implies that transmission plus reflection amount to a value of two unit fluxes (corresponding to the incidence from modes $p_{1}$ and $p_{2}$ ) is only fulfilled by the complete calcula-

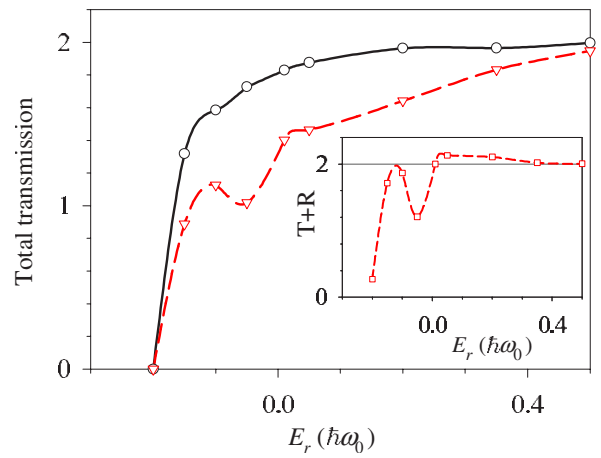

FIG. 8. (Color online) Transmission for left incidence in modes $p_{1}$ and $p_{2}$ on the potential step when $\alpha=\hbar \omega_{0} \ell_{0}$ and $E_{l}=0.93 \hbar \omega_{0}$. The step height for each value of $E_{r}$ is $V_{0}=E_{l}-E_{r}$. Circles (solid line) are the complete result while triangles (long-dashed) totally neglect the evanescent modes. Transmission plus reflection is exactly two in the complete calculation and is shown in the inset when evanescent modes are neglected. The deviation from the exact value shows the relevance of the evanescent mode contributions.

tion. Neglecting the evanescent modes leads to a violation of this balance, as shown by the inset in Fig. 8. Deviations are greater near the propagation threshold where, indeed, it is expected that evanescent modes are more important.

\section{CONCLUSIONS}

Evanescent states along a quasi-one-dimensional channel couple with the states of transverse motion when spin-orbit (Rashba) interaction is active. This greatly complicates the determination of the evanescent state wave numbers and wave functions since they do not obey a standard Hermitian eigenvalue problem. We have devised an algorithm based on the requirement that the transverse spinor wave functions fulfill boundary conditions, yielding a set of linear equations that is solvable for any complex wave number. Physically acceptable wave numbers are those having a continuous derivative at an arbitrarily chosen matching point.

Evanescent modes in Rashba wires are characterized by complex wave numbers, having real and imaginary parts. Due to symmetry, one can invert signs of either $\operatorname{Re}(k)$ or $\operatorname{Im}(k)$, or both, and still obtain physically valid wave numbers. When decreasing the energy the wire modes evolve in the following way.

(a) At high energies the mode is propagating.

(b) When the energy crosses the propagation threshold the mode becomes evanescent, with a continuous evolution of $\operatorname{Re}(k)$, while $\operatorname{Im}(k)$ suddenly starts to grow from zero.

(c) In the limit of weak Rashba coupling $\operatorname{Re}(k)$ for evanescent modes is constant and coincides with $k_{R}$, the propagation wave number at threshold, while $\operatorname{Im}(k)$ is given by Eq. (2), the result of uncoupled transverse and longitudinal motions.

(d) For intense Rashba couplings a complicated evolution of $\operatorname{Re}(k)$ and $\operatorname{Im}(k)$ with energy is observed.

A specific example illustrating the relevance of evanescent states has been solved, namely the case of a potential step in a wire with intense spin-orbit coupling. Density ac- 
cumulations at the edge, as well as distributions of spin magnetization have been obtained. Evanescent states are necessary to fulfill the boundary conditions at the step edge and maintain flux conservation. A remarkable feature of the nontrivial evanescent $\operatorname{Re}(k)$ 's is that maximal densities can be located on the evanescent mode side. We have also analyzed the case when transport to the right is allowed, finding an important contribution of the evanescent states to the transmissions.

To summarize, the evanescent modes obtained in this work allow the investigation of spin and density distributions around inhomogeneities and at interfaces in quantum wires with extended spin-orbit coupling, a condition met in many spintronic devices.

\section{ACKNOWLEDGMENTS}

This work was supported by Grant No. FIS2005-02796 (MEC) and the Spanish "Ramón y Cajal" program.

\section{APPENDIX}

In this Appendix we detail the matching conditions corresponding to the potential step in a quantum wire with Rashba interaction discussed in Sec. IV C. The general wave function is given by a superposition of modes, each one characterized by a transverse wave function and wave number $\left\{\phi_{n}(y, \eta), k_{n}\right\}$. The potential step $V_{0} \Theta(x)$ is such that with the given energy $E<V_{0}$ for left incident electrons only evanescent modes can survive in the right side. Let us distinguish the following three sets of states: $\left\{\phi_{n}, k_{n}\right\}$, the propagating modes incident from $x<0 ;\left\{\widetilde{\phi}_{n}, \widetilde{k}_{n}\right\}$, reflected propagating and evanescent modes, the latter vanishing for $x \rightarrow-\infty$ [with negative $\operatorname{Im}(k)]$; and $\left\{\hat{\phi}_{n}, \hat{k}_{n}\right\}$, evanescent modes vanishing for $x \rightarrow+\infty$. The most general state then reads

$$
\begin{aligned}
& \Psi(x, y, \eta) \\
& \quad= \begin{cases}\sum_{n} a_{n} \phi_{n}(y, \eta) e^{i k_{n} x}+\tilde{\sum}_{n} b_{n} \widetilde{\phi}_{n}(y, \eta) e^{i \tilde{k}_{n} x} & \text { if } x<0, \\
\hat{\sum}_{n} c_{n} \hat{\phi}_{n}(y, \eta) e^{i \hat{k}_{n} x} & \text { if } x>0,\end{cases}
\end{aligned}
$$

where $a_{n}, b_{n}$, and $c_{n}$ are the usual incidence, reflection, and transmission amplitudes.

The matching conditions at $x=0$ require continuity of the wave function and its $x$-derivative,

$$
\begin{gathered}
\sum_{n} a_{n} \phi_{n}(y, \eta)+\tilde{\sum}_{n} b_{n} \widetilde{\phi}_{n}(y, \eta)=\tilde{\sum}_{n} c_{n} \hat{\phi}_{n}(y, \eta), \\
\sum_{n} a_{n} k_{n} \phi_{n}(y, \eta)+\tilde{\sum}_{n} b_{n} \widetilde{k}_{n} \widetilde{\phi}_{n}(y, \eta)=\tilde{\sum}_{n} c_{n} \hat{k}_{n} \hat{\phi}_{n}(y, \eta) .
\end{gathered}
$$

Equations (A2) and (A3) must determine the reflection and transmission coefficients, $b_{n}$ and $c_{n}$, in terms of the incident ones $a_{n}$ and they amount to a linear system of two equations for each value of $y$ and $\eta$. Since we have in principle an infinite set of $y$ values, one needs also an infinite set of amplitudes $b_{n}$ and $c_{n}$ in order to have as many equations as unknowns. However, at a given energy the number of propagating states is always finite, which is illustrating the fact that one needs to include the evanescent modes, also an infinite set, to fulfill Eqs. (A2) and (A3) for arbitrary y. In practice we truncate the sums over evanescent states $\widetilde{\Sigma}_{n}$ and $\hat{\Sigma}_{n}$ and project Eq. (A2) on the set $\left\{\widetilde{\phi}_{n}\right\}$ and Eq. (A3) on $\left\{\hat{\phi}_{n}\right\}$. The resulting linear system reads

$$
\begin{gathered}
\tilde{\Sigma}_{n}[\widetilde{\phi} \widetilde{\phi}]_{m n} b_{n}-\hat{\Sigma}_{n}[\widetilde{\phi} \hat{\phi}]_{m n} c_{n}=-\sum_{n}[\widetilde{\phi} \phi]_{m n} a_{n}, \\
\tilde{\sum}_{n} \tilde{k}_{n}[\hat{\phi} \tilde{\phi}]_{m n} b_{n}-\hat{\Sigma}_{n} \hat{k_{n}}[\hat{\phi} \hat{\phi}]_{m n} c_{n}=-\sum_{n} k_{n}[\hat{\phi} \phi]_{m n} a_{n},
\end{gathered}
$$

where we have introduced the following notation for overlap matrices:

$$
[\widetilde{\phi} \widetilde{\phi}]_{m n}=\sum_{\eta} \int d y \widetilde{\phi}_{m}^{*}(y, \eta) \widetilde{\phi}_{n}(y, \eta)
$$

with obvious extensions for $[\widetilde{\phi} \hat{\phi}],[\widetilde{\phi} \phi],[\hat{\phi} \widetilde{\phi}],[\hat{\phi} \hat{\phi}]$, and $[\hat{\phi} \phi]$. Including enough evanescent states $\left\{\widetilde{\phi}_{n}\right\}$ and $\left\{\hat{\phi}_{n}\right\}$ the calculation converges, giving an increasingly better fulfillment of the matching conditions and of the flux conservation.

The formalism can be trivially extended to consider the case when the energy is such that propagating modes after the step $(x>0)$ are allowed. One just needs to add the corresponding propagating modes to the set $\left\{\hat{\phi}_{n}, \hat{k}_{n}\right\}$ while all remaining equations of the Appendix are unchanged.
${ }^{1}$ P. F. Bagwell, Phys. Rev. B 41, 10354 (1990).

${ }^{2}$ J. C. Barbosa and P. N. Butcher, Superlattices Microstruct. 22, 325 (1997).

${ }^{3}$ G. Usaj and C. A. Balserio, Europhys. Lett. 72, 631 (2005).

${ }^{4}$ M. Lee and C. Bruder, Phys. Rev. B 72, 045353 (2005).

${ }^{5}$ E. I. Rashba, Fiz. Tverd. Tela (Leningrad) 2, 1224 (1960) [Sov.
Phys. Solid State 2, 1109 (1960)].

${ }^{6}$ S. Datta and B. Das, Appl. Phys. Lett. 56, 665 (1990).

${ }^{7}$ Handbook of Mathematical Functions, edited by E. M. Abramowitz and I. A. Stegun (Dover, New York, 1972).

${ }^{8}$ Harwell subroutine library.

${ }^{9}$ A. V. Moroz and C. H. W. Barnes, Phys. Rev. B 60, 14272 
(1999).

${ }^{10}$ F. Mireles and G. Kirczenow, Phys. Rev. B 64, 024426 (2001).

${ }^{11}$ M. Governale and U. Zülicke, Phys. Rev. B 66, 073311 (2002).

${ }^{12}$ M. Valín-Rodríguez, A. Puente, and Ll. Serra, Eur. Phys. J. B 34, 359 (2003).
${ }^{13}$ Yu. V. Pershin, J. A. Nesteroff, and V. Privman, Phys. Rev. B 69, 121306(R) (2004).

${ }^{14}$ L1. Serra, D. Sánchez, and R. López, Phys. Rev. B 72, 235309 (2005).

${ }^{15}$ We assume $\Theta(x)=0$ for $x<0$ and $\Theta(x)=1$ for $x>0$. 